\title{
Study of the Absorption in Solar Cells with 2D Photonic Crystals
}

\author{
A. Ounanoughi, A. Hocini*, D. Khedrouche \\ Laboratoire d'Analyse des Signaux et Systèmes, Department of Electronics, \\ University Mohamed Boudiaf of M'sila, BP.166, Route Ichebilia, M'sila, 28000 Algeria
}

\begin{abstract}
In this work, we design via numerical simulation the absorption properties of silicon nanohole arrays of vertically aligned square lattice arrays with lattice constants varying from $300 \mathrm{~nm}$ to $400 \mathrm{~nm}$, using the finite difference time-domain method (FDTD). The silicon ( $\mathrm{Si}$ ) is chosen as prototype material for this study due to better understanding of the material properties and widely available optical data. The parameters for photonic crystals are optimized through computer simulations to obtain the maximum absorption and path length enhancement. We investigated the performance of the considered structure and determined the geometrical parameters that allow a better absorption.
\end{abstract}

DOI: $10.12693 /$ APhysPolA.127.1205

PACS: 42.70.Qs, 42.79.-e, 88.40.-j

\section{Introduction}

The great challenge of photovoltaics (PV) and its widespread use remains the reduction of manufacturing cost and improving the efficiency of solar cells. The production of solar cells was and is still based mainly on crystalline silicon wafers holding about $81 \%$ of the photovoltaic world market. In particular, silicon has the advantage of being fully compatible with the well-established microelectronics technology, and one possible application of optimized thin cells is the integration in chips, where the device thicknesses are limited. In such devices the etching procedures needed to realize a photonic pattern are directly comprised in the standard chip processing. In commonly used crystalline silicon solar cells the active layer thickness is a few hundreds of microns [1], and the cost is dominated by silicon processing; this cost can be reduced if the high-quality active layer thickness is reduced to a few hundreds of nanometers.

In recent years, the relative maturity of nanophotonics has enabled the emergence of various new concepts for light management. At the same time, the development of low cost micro- and nanotechnologies compatible with the patterning of wide areas has made the implementation of such nanophotonic structures feasible. Among the widespread and promising approaches are those based on surface plasmons, including ordered top- down metallic grating and bottom-up integrated metallic nanoparticles $[2,3]$. Other routes make use of non-metallic microand nanostructures; these are typically photonic crystal $(\mathrm{PhC})$ structures, but also sub-wavelength structures (SWS) which may be patterned within the absorbing layer. The use of a $\mathrm{PhC}$ structure to control the absorption in a PV solar cell was proposed in 2006 [4] where

\footnotetext{
${ }^{*}$ corresponding author; e-mail: hocini74@yahoo.fr
}

high diffraction orders of a $\mathrm{PhC}$ back reflector were used to increase the photon path within the silicon solar cell. In [5], a PhC patterned thin silicon layer structure is proposed to increase both incident light trapping and photon lifetime in the absorbing layer. The combination of such patterned absorbers with a top anti-reflection (AR) layer and back electrodes was then proposed for 1D and 2D PhCs [6, 7]. Both reports concluded an expected relative increase of the efficiency up to around $30 \%$, following the integration of a $\mathrm{PhC}$ structure within the absorbing layer. As another approach to exploit $\mathrm{PhC}$ structures, the integration of conductive inverted opals as the intermediate layer of a tandem cell was proposed and investigated [8].

In this work, we utilize photonic crystals to simulate enhanced light-trapping in silicon solar cells. We design via numerical simulation the optical properties of silicon nanohole arrays of vertically aligned square lattice arrays with lattice constants varying from $300 \mathrm{~nm}$ to $400 \mathrm{~nm}$, using the finite difference time-domain method (FDTD). The parameters for photonic crystals are optimized through computer simulations to obtain the maximum absorption and path length enhancement. We investigated the performance of the considered structure and determined the geometrical parameters (period lattice and filling factor) that allow a better absorption. A variety of structure configurations for different structure parameters have been implemented via mode solver program and we provided theoretical prediction for certain geometries which lead to good performance of solar cell.

\section{Solar cell structure}

We consider basic structures as presented in Figs. 1a, $1 \mathrm{~b}$ and $1 \mathrm{c}$. The first one consists of a Si unpatterned layer on a glass substrate, the second and the third one consists of an Si: patterned layer on a glass substrate in $1 \mathrm{D}$ and $2 \mathrm{D}$ configurations, respectively. Incident light may then be trapped in such resonances at a wavelength 
related to the parameters of the three structures. The third structure of solar cell is made by two-dimensional photonic crystal (2D PhC), with a square array of circular holes. The period is the same in the two directions of space, allowing an independence of the structure topography over the two planar spatial directions. The main parameters of the $\mathrm{PhC}$ are then $L$ (its lattice parameter), and $f f$ defined as the ratio of the air holes diameter over the period, $f f$ is then given by $\left(1-\pi d^{2} / 4 L^{2}\right), r$ being the radius of the air holes. The thicknesses of the two configurations are considered the same and equal to $100 \mathrm{~nm}$.

Design and simulation become very important before fabrication. The numerical tools used for our simulations are based on the finite difference time-domain method (FDTD) [9], it has been used to study the influence of the geometrical parameters of the structure on the absorption in the 380-750 nm wavelength range. Using the same method of simulation, we can observe the absorption of Si unpatterned layer on glass with the same thickness.

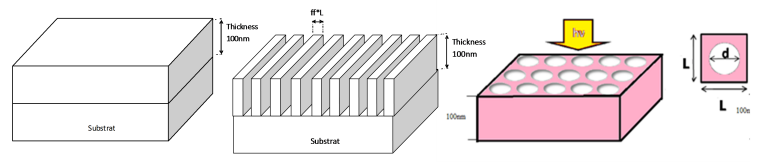

Fig. 1. (a) The structure of solar cell made by $\mathrm{Si}$ unpatterned layer on a glass substrate, (b) onedimensional photonic crystal (1D $\mathrm{PhC}$ ), with a square array of circular holes, (c) two-dimensional photonic crystal (2D PhC), with a square array of circular holes.

\section{Results and discussion}

The Rsoft [9] simulation package was used to simulate the three structures. Using Rsoft, the absorption was monitored as the wavelength was varied. The initial filling factor was fixed at $62.5 \%$ (where $d=2 r$ ); this dimension was chosen in order to maintain as large photonic band gap as possible. Using Rsoft, the absorption was monitored as the wavelength was varied.

The iteration of the simulation was repeated for a given values of the parameter $L$ (lattice parameter). Hence, the process is one in which we determine the absorption for different values of period $L=300 \mathrm{~nm}, L=380 \mathrm{~nm}$ and $L=400 \mathrm{~nm}$. We also calculate the absorption in the two other structures (unpatterned layer and 1D structure) with the same thickness $H=100 \mathrm{~nm}$.

To study the effect of patterning, the integrated absorption of one dimensional photonic crystal membrane is first optimized by varying $L$ at normal incidence of light and for fixed thickness of $100 \mathrm{~nm}$, and we compare the absorption with the unpatterned layer with the same thickness. The optimum parameters are calculated by Rsoft cad ( $L=310 \mathrm{~nm}$ ) for the $1 \mathrm{D}$ configuration. The resulting integrated absorptions are reported in Fig. 2.

It is observed that the spectra corresponding to the 1D $\mathrm{PhC}$ membrane differ from the spectrum of the unpatterend layer. In effect, the absorption in the patterned structure is higher than the unpatterned layer for all wavelengths in question. We observe also that absorption between 380 and $500 \mathrm{~nm}$ is around $50 \%$ for the two structures. In this spectral range, the extinction coefficient of silicon is so large that the absorption length which is lower than the layer thickness. Above $500 \mathrm{~nm}$, the absolute values of the absorption are reduced, due to the lower absorption coefficient of the material.

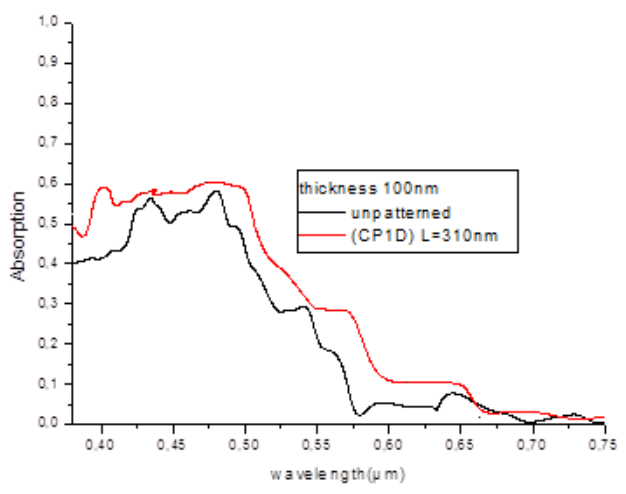

Fig. 2. Absorption spectra of the $1 \mathrm{D} \mathrm{PhC}$ structure, with a fixed filling factor of $59 \%$, and unpatterned layer with the same thickness (black continuous line).

The absorption spectra of $2 \mathrm{D} \mathrm{PhC}$ square array of circular holes with different lattice constants $(L)$ for thickness $h=100 \mathrm{~nm}$ is depicted in Fig. 3. Comparing the results of $300 \mathrm{~nm}, 380 \mathrm{~nm}$ and $400 \mathrm{~nm}$ periods, we observe that as the period increases the position of spectral absorption peaks shift toward longer wavelengths in accordance with the shifting of diffraction channels and photonic crystal modes. We can also observe that at low wavelengths, the $2 \mathrm{D} \mathrm{PhC} \mathrm{square} \mathrm{array} \mathrm{of} \mathrm{circular} \mathrm{holes}$ has an antireflection coating effect, but for high wavelengths, the absorption spectra decreases versus the extinction coefficient $k$ ( $k$ decrease rapidly). Furthermore, from the results of Fig. 3, a significant increase in absorption is observed at wavelengths above $600 \mathrm{~nm}$.

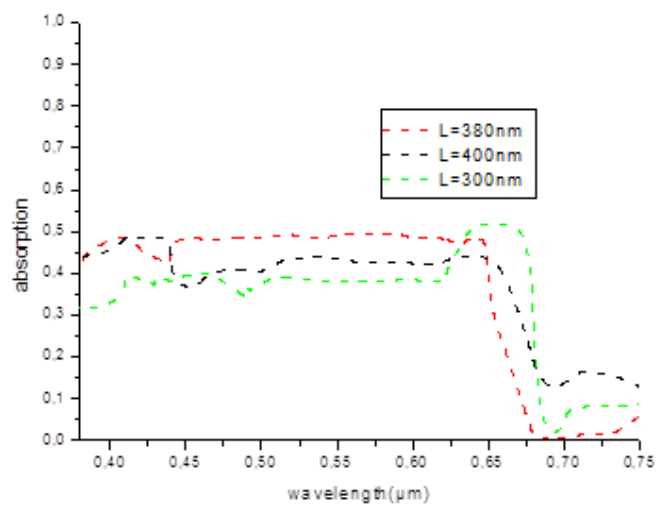

Fig. 3. Absorption spectra of $2 \mathrm{D} \mathrm{PhC}$ square array of circular holes with different lattice constants(L) for thickness $h=100 \mathrm{~nm}$ (optical simulation performed under normal incidence). 


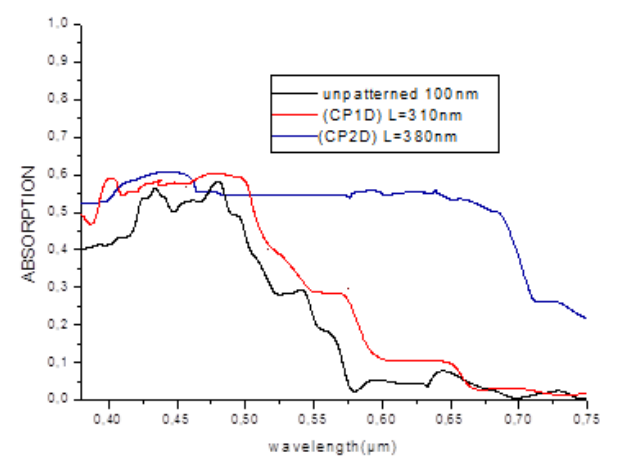

Fig. 4. Absorption spectra of 2D PC square array of circular holes with lattice constants $L=380 \mathrm{~nm}$, compared with 1D PC with lattice constants $L=310 \mathrm{~nm}$ and unpatterned (optical simulation performed under normal incidence).

Now, in order to compare the absorption in the three structures, we plot in Fig. 4 the absorption spectra of 2D PC square array of circular holes with lattice constants $L=380 \mathrm{~nm}$, compared with $1 \mathrm{D} \mathrm{PhC}$ with lattice constants $L=310 \mathrm{~nm}$ and the unpatterned layer. The optical simulations are performed under normal incidence. The spectra corresponding to the $1 \mathrm{D} \mathrm{PhC} \mathrm{mem-}$ brane strongly differ from the spectrum of the unpatterend layer. Indeed, the absorption is almost always higher irrespective of the wavelength in question. In particular, the absorption between $600-650 \mathrm{~nm}$ for the $2 \mathrm{D} \mathrm{PhC} \mathrm{configuration} \mathrm{is} \mathrm{approximately} \mathrm{six} \mathrm{times} \mathrm{larger}$ than the unstructured layer. The absorption for the $2 \mathrm{D} \mathrm{PhC} \mathrm{structure} \mathrm{becomes} \mathrm{ten} \mathrm{times} \mathrm{larger} \mathrm{in} \mathrm{the} \mathrm{range}$ of wavelength between $650-700 \mathrm{~nm}$. Consequently, we can conclude that the absorption in the $2 \mathrm{D} \mathrm{PhC}$ configuration is higher than the $1 \mathrm{D}$ and the unpatterned structures, which was corroborated by other theoretical studies $[10,11]$.

\section{Conclusions}

In this paper, the absorption properties of $1 \mathrm{D} \mathrm{PhC}$, in the patterned and unpatterned configurations, and 2D $\mathrm{PhC}$, made by silicon nanohole arrays of vertically aligned square lattice arrays, are studied and analyzed. The obtained results show an enhancement of absorption in the patterned $1 \mathrm{D} \mathrm{PhC}$ structure versus the unpatterned layer for wide range of wavelengths. Further results show that the absorption enhancement is exalted for the patterned 2D PhC, where ten times higher absorption can be reached in the range of wavelengths between 650-700 nm. Consequently, the advantages of the patterned silicon 2D Photonic Crystals make this structure a good candidate for design of efficient solar cells.

\section{References}

[1] J. Nelson in: The Physics of Solar Cells, London: Imperial College Press, 2003.

[2] H.A. Atwater, A. Polman, Nature Mater. 9, 205 (2010).
[3] R.A. Pala, J. White, E. Barnard, J. Liu, M.L. Brongersma, Adv. Mater. 21, 3504 (2009).

[4] L. Zeng, Y. Yi, C. Hong, J. Liu, N. Feng, X. Duan, L.C. Kimerling, B.A. Alamariu, Appl. Phys. Letters 89, 111111 (2006).

[5] C. Seassal, Y. Park, A. Fave, E. Drouard, E. Fourmond, A. Kaminski, M. Lemiti, X. Letartre, P. Viktorovitch, Proc. SPIE 7002, 700207 (2008).

[6] S. Zanotto, M. Liscidini, L.C. Andreani, Opt. Express 18, 4260 (2010).

[7] G. Gomard, E. Drouard, X. Letartre, X. Meng, A. Kaminski, A. Fave, M. Lemiti, E. Garcia-Caurel, C. Seassal, J. Appl. Phys. 108, 123102 (2010).

[8] A. Bielawny, J. Upping, R.B. Wehrspohn, Sol. Energy Mater. Sol. Cells 93, 1909 (2009).

[9] RSoft Design Group, FullWAVE, Inc. 200 Executive Blvd. Ossining, NY 10562.

[10] F.J. Beck, A. Polman, K.R. Catchpole, J. Appl. Phys. 105, 114310 (2009).

[11] R.A. Pala, J. White, E. Barnard, J. Liu, M.L. Brongersma, Adv. Mater. 21, 3504 (2009). 